\title{
Modificación microestructural de minerales arcillosos en ball clays de Teruel mediante tratamiento térmico
}

\author{
J.BASTIDA' ${ }^{1}$, M.T LORES ${ }^{2}$, J. DE LA TORRE, 'P. PARDO, Y A.M. LÓPEZ BUENDÍA ${ }^{3}$ \\ 'Departamento de Geología. Universidad de Valencia. ${ }^{2}$ Departamento de Ingeniería Química. Universidad de Valencia. \\ ${ }^{3}$ AIDICO (Instituto Tecnológico de la Construcción, Parque Tecnológico de Valencia)
}

\begin{abstract}
El objeto de este trabajo es estudiar la variación de límites de Atterberg en el tratamiento térmico de ball clays de la zona minera de Teruel a temperaturas inferiores a la de deshidroxilación de los filosilicatos presentes en las mismas. Dicha variación consiste en ligeras disminuciones del límite plástico y descensos más importantes en el límite líquido, y puede relacionarse, en el caso aquí considerado, con el aumento del tamaño de cristalito de los filosilicatos presentes (caolinita e illita, en este caso), hecho que se pone de manifiesto mediante un análisis microestructural simplificado por difracción de rayos $\mathrm{X}, \mathrm{y}$ mediante observación en microscopía electrónica de emisión de campo.
\end{abstract}

\section{Palabras clave: Limites de Atterberg, tratamiento térmico, ball clays, tamaño de cristalito}

\section{Microstructural modification of clay minerals in blal clays from Teruel by thermal treatment}

The aim of this paper is to analyse the influence of thermal treatments under the dehydroxilation temperature of clay minerals present in ball clays from Teruel on the Atterberg limits of these treated materials. The observed variations are slight decreases of the plastic limit and more important decreases of the liquid limit that can be related to the increase of crystallite size of the present main clay minerals (kaolinite and illite), found trough a simplified X-ray diffraction microstructural analysis and by field emision scanning electron microscopy.

Key words:

Keywords: Atterberg limits, thermal treatment, ball clays, crystallite size

\section{INTRODUCCIÓN}

La plasticidad de las arcillas humedecidas, en sentido geotécnico y también en sentido cerámico, es la aptitud a la deformarción bajo esfuerzo, sin pérdida de cohesión (1), hecho que posibilita que la arcilla pueda moldearse. Una exposición detallada de los mecanismos de fluencia plástica en cuerpos cerámicos puede hallarse en un trabajo de Bloor (2).

La deformación plástica y la resistencia a la cizalla (así como otras propiedades relacionadas) de sistemas arcilla agua varían con el contenido en agua. Así, por incorporación de agua se pasa desde un material granular sin resistencia mecánica (el polvo del material arcilloso seco), a una pasta arcillosa consistente y deformable plásticamente; de modo semejante, desde una dispersión acuosa de bajo contenido en sólidos, que se comporta como un líquido y que no presenta resistencia a la cizalla, al disminuir el contenido en agua, se pasa progresivamente a una masa plástica, que sí llega a presentarla. Atterberg hizo notar esta variación en el comportamiento mecánico del material, y puso a punto un procedimiento para su evaluación (3), basado en la determinación de : a) límite plástico : contenido en agua preciso para permitir deformación plástica , b) límite líquido: contenido en agua preciso para que el sistema se comporte como un líquido, sin presentar resistencia a la cizalla y c) índice de plasticidad: diferencia entre ambos, correspondiente al intervalo de contenidos en agua en que se tiene comportamiento plástico. Estos valores (límites e índice de plasticidad de Atterberg) han sido de gran utilidad en el estudio de suelos, desde los trabajos de Atterberg (3) y de Casagrande (4), (5) que clasificaron geotécnicamente los suelos según su ubicación en los diagramas hoy denominados de Casagrande (límite líquido frente a índice de plasticidad).

La determinación de los límites de Atterberg viene utilzándose para efectuar una valoración preliminar de plasticidad (5), (6), siendo una guía para establecer la trabajabilidad en crudo de pastas arcillosas (7), (8) y resultando de gran utilidad en la optimización de mezclas de arcillas para ladrillería (9).

La conducta plástica de arcillas y pastas cerámicas tradicionales resulta principalmente de la adsorción de agua en partículas coloidales (principalmente los filosilicatos conocidos como minerales de la arcilla)(9), de modo que las diferencias composicionales y estructurales entre los diferentes grupos de minerales de la arcilla pueden determinar sus diferencias en capacidad de adsorción de agua (10). Por otra parte, las características microestructurales (como tamaño de cristalito y distribución de tamaños de cristalitos) pueden ser efectivas asimismo, y ello será plausible comparando materiales de igual composición y diferente microestructura.

Los factores que afectan la plasticidad según límites de Atterberg en ball clays de Teruel (España) han sido considerados en un estudio previo (11), habiéndose hallado una buena correlación con composiciones mineralógicas y distribuciones granulométricas, y mala correlación con los contenidos en materia orgánica, al menos para los principales 
grupos de ball clays de la zona Ariño-Andorra, consistentes básicamente en mezclas de caolinita, illita y cuarzo, con menores cuantías de otros minerales, y contenidos variables en materia orgánica.

El tratamiento térmico a temperaturas inferiores a la de deshidroxilación de los filosilicatos presentes, aplicado a materiales arcillosos cuyos contenidos en materia orgánica son excesivos para su utilización en cocción rápida, con destino a la producción de baldosas fue objeto de una patente (12), habiendo sido estudiado a diferentes escalas en un trabajo precedente (13), en el que se apreciaba una disminución de plasticidad, pero sin llegar a ocasionar la falta de trabajabilidad por prensado.

La pérdida de plasticidad por cocción en el intervalo $500-700^{\circ} \mathrm{C}$ se ha puesto de manifiesto en diferentes trabajos $(14,15,16)$. Las variaciones en los límites Atterberg de suelos con las condiciones ambientales (hasta $40{ }^{\circ} \mathrm{C}$ ) han sido reconocidas en varios trabajos $(17,18,19)$ apreciándose variaciones significativas en el límite liquido, pero sin aportarse explicación de tales hechos.

El objeto del presente trabajo es analizar la variación observada en límites de Atterberg en ball clays de Teruel tras tratamientos térmicos por debajo de las temperaturas de deshidroxilación de los filosilicatos arcillosos

En el extenso capítulo dedicado a la deshidratación de los minerales arcillosos, en una obra de referencia como es la monografía de Grim (10), se pone de manifiesto que para illita y caolinita no existen transformaciones de fase previas a la deshidroxilación, apreciándose únicamente disminuciones en los contenidos en agua adsorbida y absorbida. Los cambios implicados en la variación de los límites de Atterberg, en el intervalo de temperaturas aludido (por debajo de la temperatura de deshidroxilación de illita y caolinita) deben pues corresponder principalmente a modificaciones microestructurales. Al efecto de comprobarlo, se ha llevado a cabo un análisis microestructural simplificado.

La influencia de la temperatura en la microestructura de los filosilicatos, como factor determinante en la "cristalinidad" "anchura de Scherrer" de las reflexiones 001 de la illita es un hecho bien establecido en la literatura geológica desde la inicial utilización de dicha anchura como índice de "cristalinidad" de Kubler, con gran número de referencias discutidas por ejemplo en la recopilación de dicho autor (20), así como en la monografía de Frey sobre metamorfismo de baja temperatura (21), con posteriores interpretaciones en términos de crecimiento cristalino (22). La utilización del término de "cristalinidad" con diferente sentidos condujo a recomendaciones (23) por parte de AIPEA (Asociación Internacional para el Estudio de las Arcillas). En el trabajo de

Clausell (24) sistematizó la influencia de las condiciones genéticas en las anchuras de las reflexiones 001 de caolinita, en caolines del Macizo Ibérico.

\section{ESTUDIOS PREVIOS Y MATERIALES}

Se han estudiado algunas ball clays de Teruel seleccionadas entre un conjunto cuya composición mineralógica fue objeto de un estudio ya aludido (11), en el que se halló una notable homogeneidad composicional. La composición mineralógica y granulométrica de los materiales, fueron reconocidas como principales factores determinantes de variación en los límites de Atterberg: siendo el índice de plasticidad influido principalmente por la distribución granulométrica mientras que el límite líquido era afectado preferentemente por la composición mineralógica. La totalidad de las muestras se representaron en un diagrama de Casagrande (límite líquido frente a índice de plasticidad) y se puso de manifiesto la correlación entre valores de límite plástico y límite líquido $\left(R^{2}\right.$ $=0.8568)$.

Los datos de las Tablas I, II, III y IV corresponden respectivamente a la composición química, análisis racional (composición mineralógica obtenida de datos químicos), composición mineralógica (obtenida por difracción de rayos $\mathrm{X})$ y otras propiedades (color, granulometría, pérdida de peso a $500^{\circ} \mathrm{C}$, contenido en $\mathrm{C}$ orgánico y límites de Atterberg) de los materiales aquí estudiados, y proceden de (11), trabajo anterior en el que se detallaron las técnicas analíticas y se

TABLA I. ANÁLISIS QUÍMICO. DATOS EXTRAIDOS DE (11)

\begin{tabular}{|l|c|c|c|c|c|c|}
\hline & PT-11 & PT-13 & PT-14 & PT-15 & PT-16 & PT-26 \\
\hline $\mathrm{SiO} 2$ & 63,10 & 68,40 & 48,1 & 39,20 & 40,8 & 59,40 \\
\hline $\mathrm{Al2O} 3$ & 19,00 & 18,50 & 29,3 & 28,60 & 27,1 & 24,70 \\
\hline $\mathrm{Fe} 2 \mathrm{O} 3$ & 4,39 & 1,56 & 3,56 & 11,40 & 7,49 & 0,99 \\
\hline $\mathrm{CaO}$ & 0,24 & 0,54 & 0,38 & 2,98 & 3,51 & 0,06 \\
\hline $\mathrm{MgO}$ & 0,86 & 0,56 & 0,61 & 0,62 & 0,78 & 0,54 \\
\hline $\mathrm{Na2O}$ & 0,17 & 0,15 & 0,06 & 0,07 & 0,57 & 0,32 \\
\hline $\mathrm{K} 2 \mathrm{O}$ & 3,00 & 2,42 & 0,8 & 1,05 & 0,44 & 2,29 \\
\hline $\mathrm{TiO} 2$ & 0,97 & 0,99 & 1,88 & 1,27 & 1,57 & 1,55 \\
\hline $\mathrm{MnO}$ & 0,04 & 0,03 & 0,03 & 0,05 & 0,05 & 0,03 \\
\hline $\mathrm{P} 2 \mathrm{O} 5$ & 0,06 & 0,11 & 0,06 & 0,09 & 0,06 & 0,09 \\
\hline $\mathrm{Cr} 2 \mathrm{O} 3$ & 0,01 & 0,01 & 0,01 & 0,01 & 0,01 & 0,01 \\
\hline $\mathrm{PC}$ \\
$\left(1050{ }^{\circ} \mathrm{C}\right)$
\end{tabular}

TABLA II. ANÁLISIS RACIONAL: CONSTITUYENTES MINERALOGICOS A PARTIR DE COMPOSICION QUÍMICA. DATOS EXTRAIDOS DE (11)

\begin{tabular}{|l|c|c|c|c|c|c|}
\hline & PT-11 & PT-13 & PT-14 & PT-15 & PT-16 & PT-26 \\
\hline Illita & 5,64 & 20,5 & 6,8 & 8,9 & 3.7 & 19,4 \\
\hline Caolinita & 23,4 & 26,9 & 67,6 & 63,8 & $65, .0$ & 43,7 \\
\hline Cuarzo & 40,7 & 46,6 & 13,6 & 5,5 & 8,9 & 30,3 \\
\hline
\end{tabular}

TABLA III. ANÁLISIS MINERALÓGICO POR DIFRACCION DE RAYOS X. (ESTIMACIÓN SEMICUANTITATIVA POR EL MÉTODO DE INTENSIDADES DE REFERENCIA) DATOS EXTRAIDOS DE (11)

\begin{tabular}{|c|c|c|c|c|c|c|}
\hline$\%$ & PT-11 & PT-13 & PT-14 & PT-15 & PT-16 & PT-26 \\
\hline Filosilicatos & 54 & 42 & 90 & 92 & 88 & 68 \\
\hline Cuarzo & 40 & 54 & 9,5 & 1,4 & 8,2 & 29 \\
\hline Calcita & 2,2 & 1,7 & $<0,1$ & 2,8 & 2,2 & 1,4 \\
\hline Hematites & 0,6 & 1,4 & $<0,1$ & 2 & 0,9 & $<0,1$ \\
\hline Feldespatos & 2 & 1 & 0 & $<0,1$ & 0 & 0,4 \\
\hline Siderita & 1,3 & $<0,1$ & $<0,1$ & 0,2 & $<0,1$ & 0,5 \\
\hline Pirita & 0,2 & $<0,1$ & 0 & 1,5 & $<0,1$ & $<0,1$ \\
\hline Dolomita & 0,2 & 0,2 & $<0,1$ & $<0,1$ & 0,4 & 0,15 \\
\hline Clorita & 0 & 0,6 & 0 & 0 & 6 & 0 \\
\hline Illita & 30 & 15 & 3,9 & 3 & 0,3 & 22 \\
\hline Caolinita & 24 & 26 & 86 & 89 & 82 & 22 \\
\hline Esmectita & $<0,1$ & 0,2 & 0,1 & $<0,1$ & $<0,1$ & $<0,1$ \\
\hline
\end{tabular}


TABLA IV. ALGUNAS CARACTERÍSTICAS PETROGRÁFICAS Y LÍMITES DE ATTERBERG DE LAS MUESTRAS SIN TRATAR. DATOS EXTRAIDOS DE (11)

\begin{tabular}{|c|c|c|c|c|c|c|c|c|}
\hline & COLOR & $\%$ arcilla & $\%$ limo & $\begin{array}{c}\% \text { pérdida a } \\
500{ }^{\circ} \mathrm{C}\end{array}$ & $\%$ C org & LL & LP & IP \\
\hline PT-11 & blanco & 33,3 & 38 & 4,59 & 0,17 & 33,5 & 18,9 & 14,6 \\
\hline PT-13 & marrón & 29 & 40,66 & 3,69 & 0,05 & 31 & 17,9 & 13,1 \\
\hline PT-14 & gris & 49,39 & 22,99 & 11,1 & 0,96 & 70 & 27,7 & 42,3 \\
\hline PT-15 & salmón & 41 & 26,33 & 9,91 & 0,05 & 55 & 29,9 & 25,1 \\
\hline PT-16 & beige & 49 & 20 & 11.32 & 1.18 & 67 & 32,1 & 35 \\
\hline PT-26 & beige & 71 & 20,67 & 6,57 & 0,29 & 50,3 & 26,4 & 23,9 \\
\hline
\end{tabular}

compararon los diferentes resultados, en un conjunto más amplio de muestras.

En el presente trabajo, se efectuan tratamientos térmicos y determinaciones de límites de Atterberg de las muestras tratadas utilizando las muestras PT13, PT14, PT15 y PT16. Se utiliza un análisis microestructural simplificado de los filosilicatos en muestras tratadas y sin tratar, para explicar la evolución de los límites de Atterberg con el tratamiento.

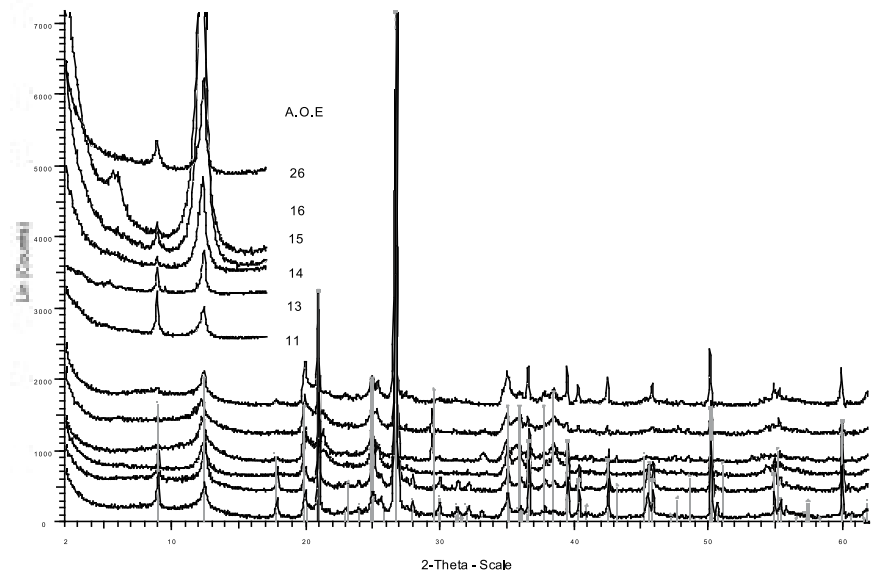

Fig. 1- Difractogramas de las muestras totales (debajo) y de los agregados orientados de fracción arcilla, tratados con etilenglicol (el orden de representación es el indicado para los agregados orientados). Líneas indicativas de fichas ICDD: cuadro vertical: illita; cuadro sobre vértice: caolinita; círculo: cuarzo; triángulo: calcita. Escala horizontal: ${ }^{\circ}(2 \theta) \mathrm{Cu} \mathrm{K} \alpha_{1}$

La Fig. 1 presenta los difractogramas de muestra de polvo total de las muestras seleccionadas, incluyendo asimismo los correspondientes difractogramas de agregados orientados etilenglicolados (A.O.E). Dichos agregados, se prepararon por contacto del agregado seco de fracción arcilla (obtenido por decantación de suspensión de fracción arcilla sobre porta de vidrio y secado) con papel de filtro impregnado con etilenglicol, durante 12 horas, según los procedimientos mineralógicos descritos en (11). Nótese la presencia de pequeñas cuantías de esmectita en la muestra PT13.

La Fig. 2 ilustra la variabilidad composicional de los materiales atendiendo a su posición en diagrama triangular de constituyentes mayoritarios con datos de análisis racional (estimación de constituyentes mineralógicos a partir de datos de composición química).

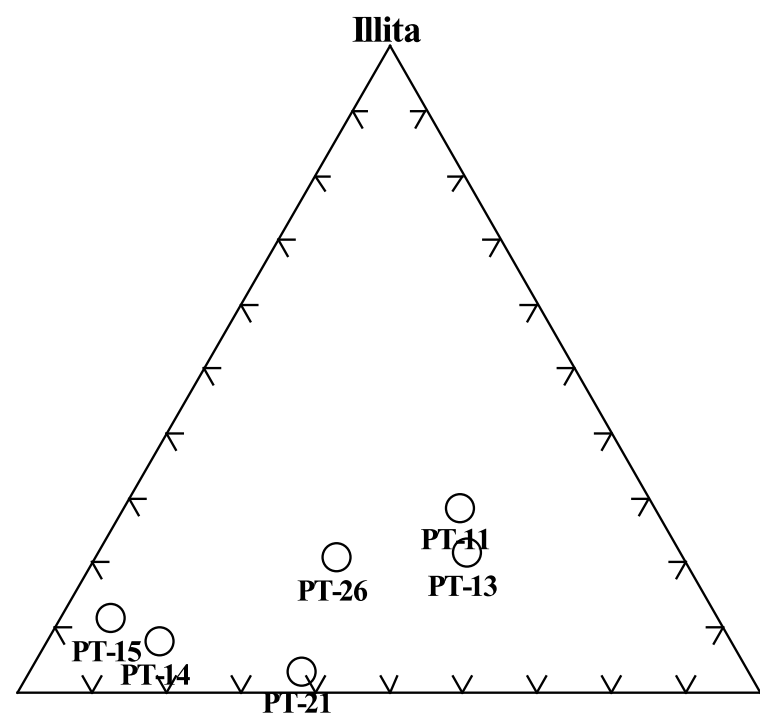

Caolinita

Cuarzo

Fig. 2- Composición mineralógica de muestras estudiadas (constituyentes mayoritarios).

\section{MÉTODOS EXPERIMENTALES}

\subsection{Tratamiento térmico.}

Los tratamientos en muestras de material pulverizado se efectuaron en un horno rotatorio descrito (como dispositivo B) en un trabajo precedente (12), consistente básicamente en un cilindro de acero calorifugado, de $9 \mathrm{~cm}$ de diámetro interno y $15 \mathrm{~cm}$ de longitud, en el que se procesó polvo del material previamente desecado durante 12 horas a $110^{\circ} \mathrm{C}$. En el horno rotatorio (a una velocidad de $60 \mathrm{rpm}$, con un caudal de 450 1 / hora de aire de aire a una presión parcial de oxígeno de 0.21 atm procedente del precalentador) se efectuó la calefacción a las diferentes temperaturas, y tiempos de cada experiencia, procediéndose en modo discontinuo, y descargando el horno al final de cada experiencia. En el material descargado del horno en cada experiencia, se practicaron medidas de límites de Atterberg, como se describe en el epígrafe siguiente.

Las determinaciones microestructurales por DRX, se practicaron en agregados orientados de fracción arcilla, calentadas en horno mufla, con las mismas permanencias en horno descritas en el párrafo anterior para las experiencias en horno rotatorio.

\subsection{Límites de Atterberg.}

Los límites de Atterberg, límite plástico (PL), y límite liquido (LL) se determinaron según los procedimientos descritos en las normas NLT105 y NLT106, correspondientes a la norma ASTM D423, (25). A continuación, se describe lo esencial del procedimiento, sin entrar en los detalles, que quedan minuciosamente recogidos en las normas.

Para la obtención del límite plástico se determina el contenido en humedad de una pasta plástica arcilla- agua moldeada en forma cilíndrica, cuando pierde la cohesión como consecuencia de un esfuerzo mecánico suave ejercido por la palma de la mano del operador, teniendo un diámetro aproximado especificado en la norma $(3 \mathrm{~mm})$. Cuando el contenido de humedad de la pasta es muy superior al límite plástico, se consiguen cilindros de diámetro muy inferior al 
especificado, y bajo la acción de la palma de la mano del operador, la pasta se deforma plásticamente sin perder la cohesión. Procediendo según la norma, con el fino cilindro se vuelve a moldear una bola que nuevamente es deformada bajo la acción de la palma de la mano (en el curso de estas operaciones se va retirando humedad, principalmente por la palma de la mano, que debe estar limpia y seca; el proceso se puede acelerar moldeando los cilindros sobre una superficie lisa de un material poroso; la norma no fija el tipo de material). La experiencia se repite varias veces hasta conseguir que la pérdida de cohesión se alcance con el diámetro especificado; en ese momento, se toman fragmentos cilíndricos y se determina su contenido en humedad, procediendo según norma.

Como se ha indicado en la introducción, el límite líquido representa el menor contenido en agua que permite que la pasta se comporte como un líquido. Para determinación de tal límite se utiliza el instrumento ideado por Casagrande (4), hoy conocido como cuchara de Casagrande. Con el material granular (en nuestro caso material arcilloso en polvo) convenientemente impregnado de agua (según se establece en la norma), se cubre del modo adecuado el receptáculo del instrumento, y sobre la superficie de la pasta húmeda, se practica un surco, de sección normalizada utilizando un acanalador (normalizado). Se estima que la pérdida de resistencia a la cizalla de la pasta se consigue, cuando el surco practicado se cierra a lo largo de una longitud mínima normalizada $(13 \mathrm{~mm})$, como consecuencia de un número establecido (concretamente, 25) de golpes del receptáculo a su base bajo la acción de una manivela (accionada manualmente o motorizada), en esas condiciones, se determina el contenido en humedad de la porción de pasta en torno al cierre del surco. Con humedad creciente de la pasta, disminuye el número de golpes preciso para ocasionar el cierre, y viceversa; el límite líquido correspondería al contenido en humedad, al producirse el cierre con 25 golpes, y según la norma se obtiene promediando (según establece la norma) valores obtenidos con porciones de pasta cerradas respectivamente entre 15 y 25 golpes, por una parte, y entre 25 y 35, por otra.

\subsection{Análisis microestructural por difracción de rayos $X$.}

Se efectuaron determinaciones de tamaño de cristalito presentes en las arcillas con distintos tratamientos térmicos, para justificar las variaciones apreciadas en los límites de Atterberg tras dichos tratamientos.

Existen datos microestructurales por difracción de rayos $X$ relativos a caolinitas de estos tipos de arcillas (26) pero no relativos a sus illitas. De acuerdo con el referido trabajo, no se aprecian deformaciones de red en la dirección de difracción [001] en las caolinitas estudiadas, con lo cual los valores de FWHM (anchura a media altura) de las reflexiones 001 dependen únicamente del tamaño de cristalito en esa dirección. Asumiendo lo mismo para la illita, se ha practicado un procedimiento simplificado de estudio de las reflexiones 001 de ambos minerales.

Los registros de difracción de rayos $X$ se practicaron en un difractómetro SIEMENS D-500, a $40 \mathrm{KV}$ y $20 \mathrm{~mA}$, controlado con un PC y trabajando bajo el sistema DIFFRAC-AT de Socabim. Los estudios microestructurales se efectuaron en registros lentos de reflexiones 001 de los filosilicatos obtenidos en los agregados orientados antes aludidos. Se utilizó radiación $\mathrm{Cu} \mathrm{K} \alpha$ con filtro de $\mathrm{Ni}$, y un sistema de ventanas grandes $1^{\circ}, 1^{\circ}, 0.25^{\circ}$, siendo las condiciones de adquisición de datos: registro a pasos de $0.025^{\circ} 2 \theta$ y tiempos de conteo de 1.5 y 4 segundos para los difractogramas (de polvo desorientado y de agregados orientados) preliminares de caracterización mineralógica, mientras que los registros lentos utilizados para análisis microestructural, en agregados orientados, se obtuvieron por conteo a pasos de $0.005^{\circ} 2 \theta$ y con tiempos de conteo (variables en función de la intensidad del pico) de 7 y 30 segundos, para las reflexiones 001 de caolinita e illita respectivamente.

El análisis de los perfiles de las reflexiones seleccionadas, se efectuó utilizando el programa PROFILE, del paquete DIFFRACAT. Los valores de anchura de los perfiles $\mathrm{CuK} \alpha_{1}$, utilizados para el cálculo de tamaño aparente de cristalito a partir de la ecuación de Scherrer (27). se obtuvieron en funciones split pseudo-Voigt (28) ajustadas a los perfiles experimentales tras sustracción de fondo obtenido por ajuste lineal a los extremos.

Los tamaños aparentes de cristalito en la dirección [001] para illita y caolinita se obtuvieron con mediciones relativas a las reflexiones 001 de illita y caolinita, utilizando las reflexiones 001 y 010 de moscovita y yeso (a $10 \AA ̊$ y 7,5 $\AA$ respectivamente) medidas respectivamente en monocristales de moscovita y yeso molidos a tamaño de grano $<40 \mu \mathrm{m}$, como patrones para obtención de las anchuras instrumentales.

\subsection{Análisis micromorfológico en FESEM.}

Con objeto de integrar los datos obtenidos por difracción en un marco micromorfológico, se practicaron observaciones mediante microscopía electrónica de barrido de emisión de campo (FESEM) a alta magnificación (entre $\times 10^{5}$ y $2 \times 10^{5}$ ) con un equipo Hitachi 4100 (trabajando a $15-30 \mathrm{KV}$, voltaje de extracción de $3.4 \mathrm{KV}$ e intensidad de $10 \mu \mathrm{A}$ ). Se utilizaron agregados orientados sobre una lámina de aluminio dispuesta paralelamente al haz de electrones, para reconocimiento micromorfológico de las laminillas de exfoliación de los filosilicatos arcillosos presentes, en una muestra de fracción arcillosa caolinítica sin tratamiento térmico, y en otra con el tratamiento a más temperatura y de mayor duración de los considerados en el análisis microestructural por difracción de rayos $X$.

\section{RESULTADOS Y DISCUSION}

\subsection{Límites de Atterberg.}

La Tabla V presenta los valores experimentales de límites de Atterberg, así como las disminuciones de los valores de LL y LP (DLL y DLP) con diferentes tratamientos térmicos.

Debe notarse que la muestra PT-13 tratada presenta una disminución de LL significativa (aunque pequeña, en comparación con las demás muestras tratadas), y una anomalía para LP, que podría derivar del hecho de que la pérdida de peso a $110{ }^{\circ} \mathrm{C}$, utilizada en la determinación del LP de la muestra sin tratar no incluyera la totalidad del agua absorbida en esmectitas, que pudiera perderse a temperatura algo superior.

\subsection{Análisis microestructural simplificado.}

Las Tablas VI y VII muestran los valores experimentales de FWHM (full width at half the maximum) para reflexiones 001 de illita y caolinita, en las muestras PT11 y PT26 sometidas a diferentes tratamientos térmicos, y los tamaños aparentes de cristalito correspondientes $\mathrm{L}\left(\mathrm{I}_{[001]}\right)$ y $\mathrm{L}\left(\mathrm{K}_{[001]}\right)$. Dichas muestras 
TABLA V. VARIACIÓN DE LÍMITES DE ATTERBERG.

\begin{tabular}{|c|c|c|c|c|c|c|c|}
\hline REF. & $\mathrm{T}\left({ }^{\circ} \mathrm{C}\right)$ & $\mathrm{t}(\mathrm{min})$ & $\mathrm{LL}$ & $\mathrm{DLL}(\%)$ & $\mathrm{LP}$ & $\mathrm{DLP}(\%)$ & $\mathrm{IP}$ \\
\hline PT-14 & - & - & 64 & - & 29,1 & - & 34,9 \\
\hline & 350 & 45 & 40 & 37,5 & 27,6 & 5,1 & 12,4 \\
\hline & 400 & 15 & 38 & 40,6 & 28,1 & 3,4 & 9,9 \\
\hline & 450 & 5 & 37 & 42,1 & 27,7 & 4,8 & 9,3 \\
\hline PT-15 & - & - & 51 & 0 & 24,2 & 0 & 26,8 \\
\hline & 450 & 5 & 32 & 37,2 & 23,8 & 1,6 & 8,2 \\
\hline PT-13 & - & - & 28 & - & 16,6 & - & 11,4 \\
\hline & 450 & 5 & 26 & 7,1 & 17,9 & $-7,8$ & 8,1 \\
\hline PT-16 & - & 0 & 57 & - & 30,1 & - & 26,9 \\
\hline & 350 & 20 & 46 & 19,2 & 29,8 & 0,9 & 16,2 \\
\hline & & 180 & 44 & 22,8 & 29,4 & 2,3 & 14,6 \\
\hline & 400 & 10 & 42 & 26,3 & 29,4 & 2,3 & 12,6 \\
\hline & & 120 & 40 & 29,8 & 29,2 & 2,9 & 10,8 \\
\hline & 450 & 7 & 39 & 31,5 & 27,7 & 7,9 & 11,3 \\
\hline & & 30 & 37 & 35,0 & 26,9 & 10,6 & 10,1 \\
\hline & 500 & 5 & 36 & 36,8 & 29,6 & 1,6 & 6,4 \\
\hline
\end{tabular}

TABLA VI. FWHM Y TAMAÑOS APARENTES DE CRISTALITO EN ILLITA, CON DISTINTOS TRATAMIENTOS

\begin{tabular}{|c|c|c|c|c|}
\hline & \multicolumn{2}{|c|}{ Tratamiento } & FWHM & $\mathrm{L}\left(\mathrm{I}_{[001]}\right)$ \\
\hline \multirow[t]{4}{*}{ REF. } & $\mathrm{t}$ (min.) & $\mathrm{T}\left({ }^{\circ} \mathrm{C}\right)$ & $(2 \theta)$ & $(\AA ̊)$ \\
\hline & \multicolumn{2}{|c|}{ Sin } & 0,507 & 178,6 \\
\hline & & 200 & 0,507 & 198,6 \\
\hline & 5 & 350 & 0,480 & 189,1 \\
\hline \multirow[t]{7}{*}{ PT-11 } & & 450 & 0,411 & 222,9 \\
\hline & & 200 & 0,507 & 178,6 \\
\hline & 30 & 350 & 0,460 & 197,8 \\
\hline & & 450 & 0,409 & 224,1 \\
\hline & \multicolumn{2}{|c|}{ Sin } & 1,154 & 77,0 \\
\hline & & 200 & - & - \\
\hline & 5 & 350 & 0,906 & 82,7 \\
\hline \multirow[t]{4}{*}{ PT-26 } & & 450 & 0,549 & 164,4 \\
\hline & & 200 & 0,805 & 111,0 \\
\hline & 30 & 350 & 0,607 & 148,2 \\
\hline & & 450 & 0,457 & 199,2 \\
\hline
\end{tabular}

TABLA VII. FWHM Y TAMAÑOS APARENTES DE CRISTALITO EN CAOLINITA, CON DISTINTOS TRATAMIENTOS

\begin{tabular}{|c|c|c|c|c|}
\hline & \multicolumn{2}{|c|}{ Tratamiento } & FWHM & $\mathrm{L}_{\left(\mathrm{K}_{[001]}\right)}$ \\
\hline REF. & $\mathrm{t}(\mathrm{min})$. & $\mathrm{T}\left({ }^{\circ} \mathrm{C}\right)$ & $(2 \theta)$ & $(\AA)$ \\
\hline & \multicolumn{2}{|c|}{ Sin } & 0,413 & 177,9 \\
\hline & & 200 & 0,413 & 181,1 \\
\hline & 5 & 350 & 0,411 & 192,1 \\
\hline PT-26 & & 450 & 0,343 & 227,6 \\
\hline & & 200 & 0,413 & 180,6 \\
\hline & 30 & 350 & 0,411 & 200,6 \\
\hline & & 450 & 0,334 & 228,3 \\
\hline
\end{tabular}

son representativas de los valores extremos de FWHM en reflexiones 001 de las illitas de las arcillas consideradas en el trabajo antes aludido (11). La muestra 26 presenta también una caolinita con un valor de FWHM representativo de la mayoría de muestras estudiadas.

La Fig. 3 muestra los perfiles 001 de illita de la muestra PT11 y la Fig. 4, de perfiles 001 de caolinita de la muestra PT26. Se aprecian aumentos de intensidad con aumentos de tiempos y temperaturas de tratamiento en la Fig. 3, pero no en la Fig. 4, donde se aprecia decrecimiento de intensidad con el tiempo a $450{ }^{\circ} \mathrm{C}$, aunque siempre hay decrecimiento en los valores de FWHM y por consiguiente aumentos en los tamaños aparentes de cristalito, que son mayores a las temperaturas mayores $\left(350\right.$ y $\left.450^{\circ} \mathrm{C}\right)$ y con los mayores

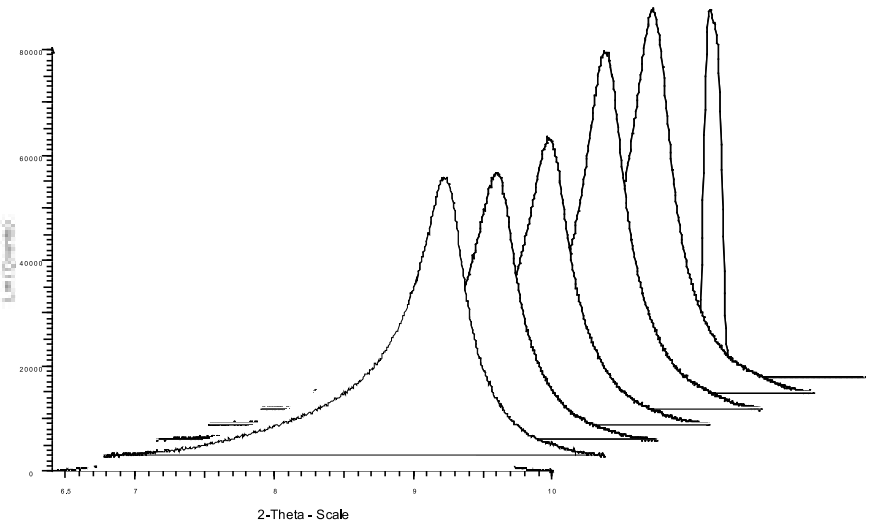

Fig. 3- Registros difractométricos lentos (paso de $0.005^{\circ}$, tiempo de conteo: $30 \mathrm{sg}$ ) de los picos 001 de illita de muestra PT-11. De delante hacia atrás: sin tratar, a $200^{\circ} \mathrm{C}(5 \mathrm{~min}),. 200^{\circ} \mathrm{C}(30 \mathrm{~min}),. 350^{\circ} \mathrm{C}(5 \mathrm{~min}$.), $350^{\circ} \mathrm{C}(30 \mathrm{~min}),. 450^{\circ} \mathrm{C}(5 \mathrm{~min}),. 450^{\circ} \mathrm{C}(30 \mathrm{~min}$.), Patrón. Escala horizontal: ${ }^{\circ}(2 \theta) \mathrm{Cu} \mathrm{K \alpha}$

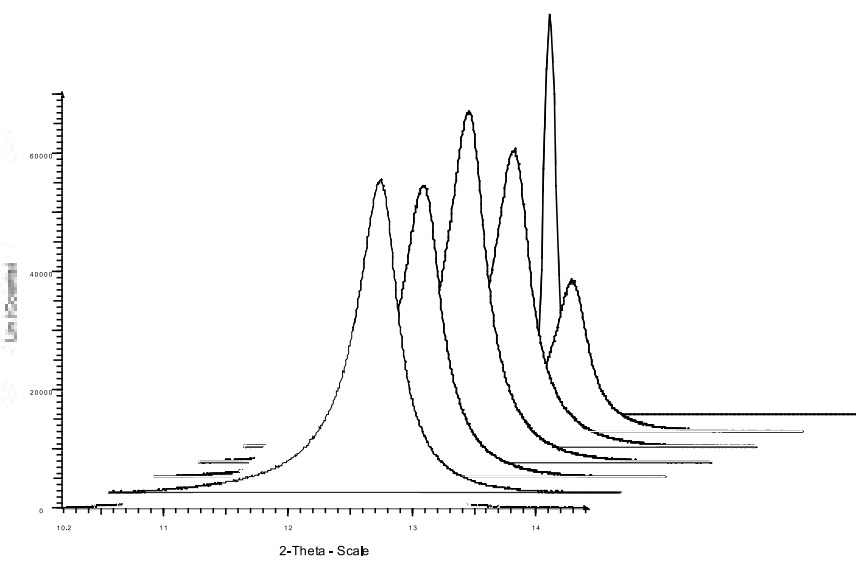

Fig. 4- Registros difractométricos lentos (paso de $0.005^{\circ}$, tiempo de conteo, $7 \mathrm{sg}$ ) de los picos 001 de caolinita de muestra PT-26. De delante hacia atrás: sin tratar, $200^{\circ} \mathrm{C}(30 \mathrm{~min}),. 350^{\circ} \mathrm{C}(5 \mathrm{~min}),. 350^{\circ} \mathrm{C}(30$ $\min$.), $450^{\circ} \mathrm{C}$ (5 min.), $450^{\circ} \mathrm{C}$ (30 min.), Patrón. Escala horizontal: ${ }^{\circ}(2 \theta)$ $\mathrm{Cu} \mathrm{K \alpha} \alpha_{1}$

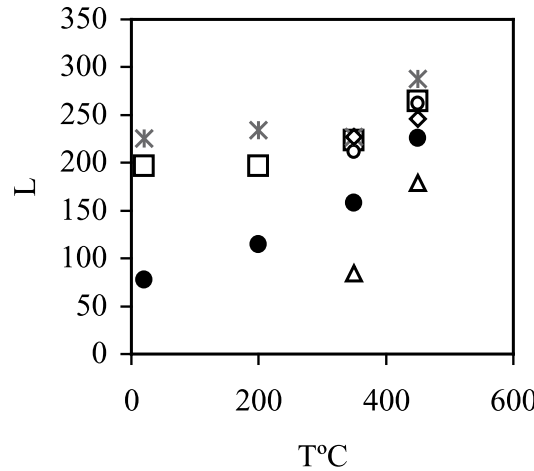

$\square \mathrm{L}(30 \mathrm{~min}) \mathrm{PT} 11$

- L (5 min) PT11

- L (30 min) PT26

$\Delta \mathrm{L}(5 \mathrm{~min}) \mathrm{PT} 26$

$* \operatorname{LK}(5 \mathrm{~min})$

$\diamond \mathrm{L} \mathrm{K}(30 \mathrm{~min})$

Fig. 5- Evolución con la temperatura de tamaños de cristalito (en Å) de caolinita (LK) de la muestra PT26 y de illita (L) en las muestras indicadas. 
tiempos de tratamiento como se recoge en las Tablas VI y VII. Las variaciones de tamaño aparente de cristalito se representan en la Fig. 5.

\subsection{Observaciones micromorfológicas en FESEM.}

Debe notarse que las variaciones registradas en el análisis precedente no son tan evidentes para caolinita (Fig.5), pero la evolución microestructural mostrada en la Tabla VII se ha puesto de manifiesto para los extremos, en caolinita que es el caso más desfavorable, mediante observación micromorfológica por microscopía electrónica de emisión de campo (FESEM). Las imágenes de FESEM obtenidas con la metodología expuesta, tomando en consideración el hábito normal de la caolinita (29), permiten reconocer con facilidad una agregación de laminillas paralelas a las caras de mayor desarrollo que son las (001), y cuyo espesor se puede medir cuando se disponen paralelamente al haz de electrones; dichas laminillas, en su agregación, pueden dar lugar a superficies planas o rugosas tomando como referencia tanto planos paralelos a [001] como planos paralelos a (001); este segundo caso se hace patente cuando la inclinación de las laminillas respecto al haz es suficientemente grande, entonces, se pueden reconocer caras (001) de muy diferente desarrollo, lo cual es muy frecuente en caolinita, y determina rugosidad en planos paralelos a (001), y que eventualmente es consecuencia de crecimiento helicoidal, como ha sido puesto de manifiesto por Zbik y Smart (30), autores que identifican como cristalitos las laminillas de FESEM a alta magnificación (como en nuestro caso) y miden escalones de crecimiento mediante microscopía de fuerza atómica.

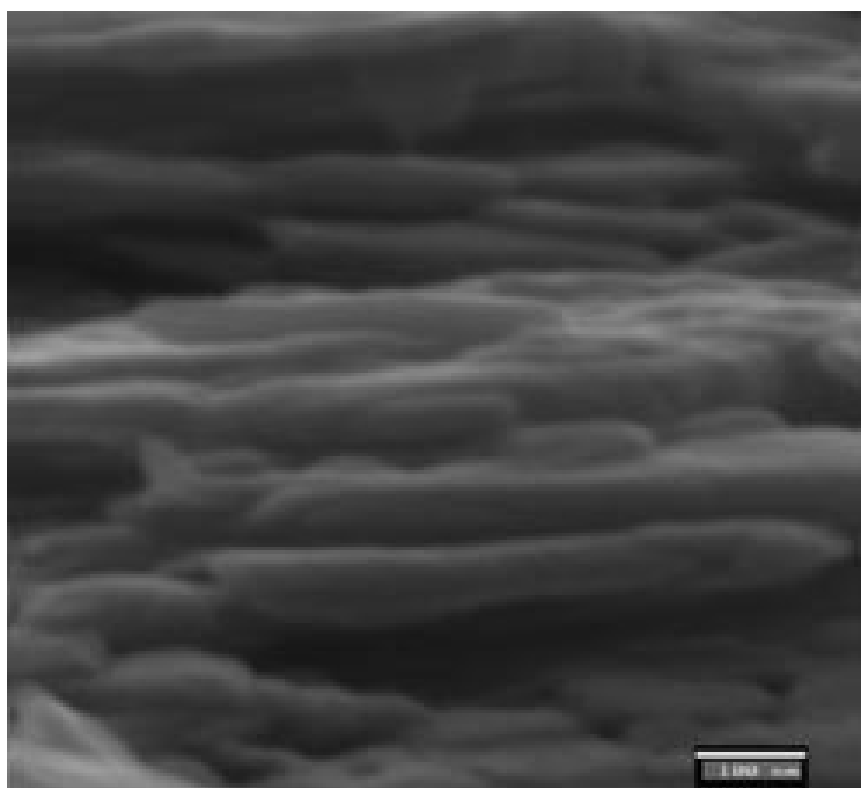

Fig. 6- Imagen en FESEM de caolinita sin tratamiento. Longitud de la barra, $100 \mathrm{~nm}$.

Las imágenes, Fig.6 y Fig.7, corresponden respectivamente a muestras de fracción arcilla predominantemente caolinítica a temperatura ambiente y a $450^{\circ} \mathrm{C}(30 \mathrm{~min})$. En el segundo caso muestran una mayor frecuencia de los espesores superiores a $50 \mathrm{~nm}$ (marca g) que pone de manifiesto el espesamiento de los cristalitos apreciado mediante difracción.

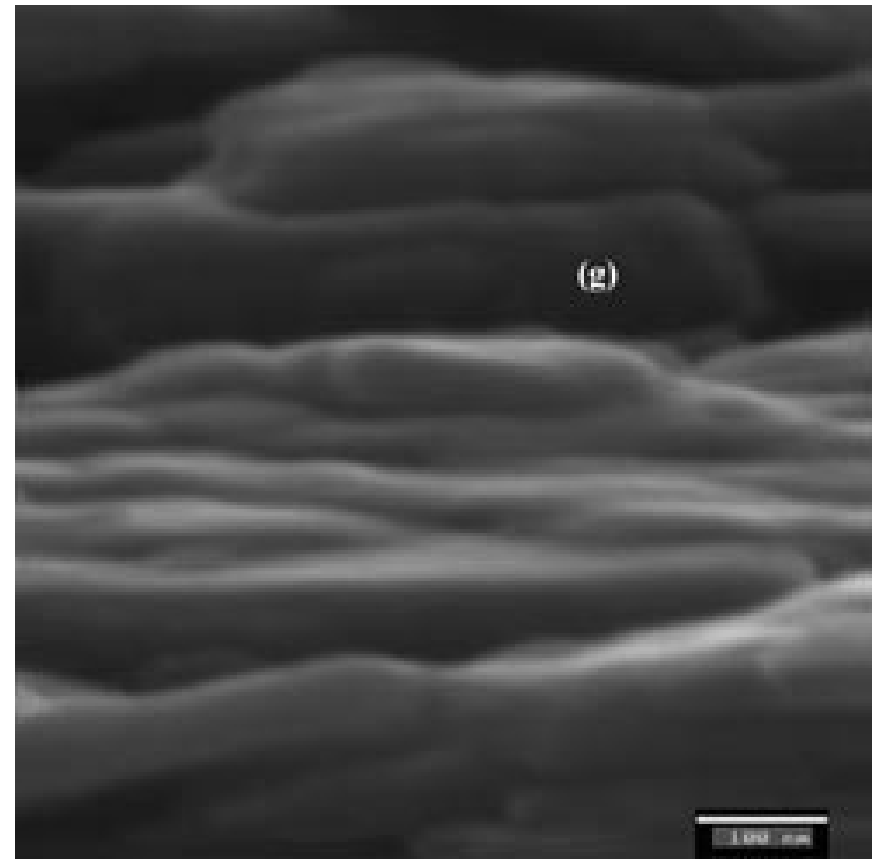

Fig. 7-Imagen en FESEM de caolinita, calentada 60 minutos a $450{ }^{\circ} \mathrm{C}$. Longitud de la barra, $100 \mathrm{~nm}$. La muestra presenta mayor frecuencia de cristalitos de espesor superior a $55 \mathrm{~nm}$ (como el marcado como g, y el situado encima).

Las imágenes muestran además la disminución de rugosidad debida a ondulaciones respecto a planos paralelos a (001), en relación con las laminillas de menor espesor (que son las predominantes en ambas muestras. El reconocimiento de imágenes con orientaciones diferentes a las mostradas, permite apreciar también disminución de rugosidad en planos paralelos a [001], pues disminuye el número de unidades que pueden tener diferente desarrollo (no siempre se da tal caso) en el mismo volumen.

\subsection{Discusión.}

Considerando los tamaños de cristalito de caolinita e illita obtenidos en experimentos con la muestra 26 , se ha calculado

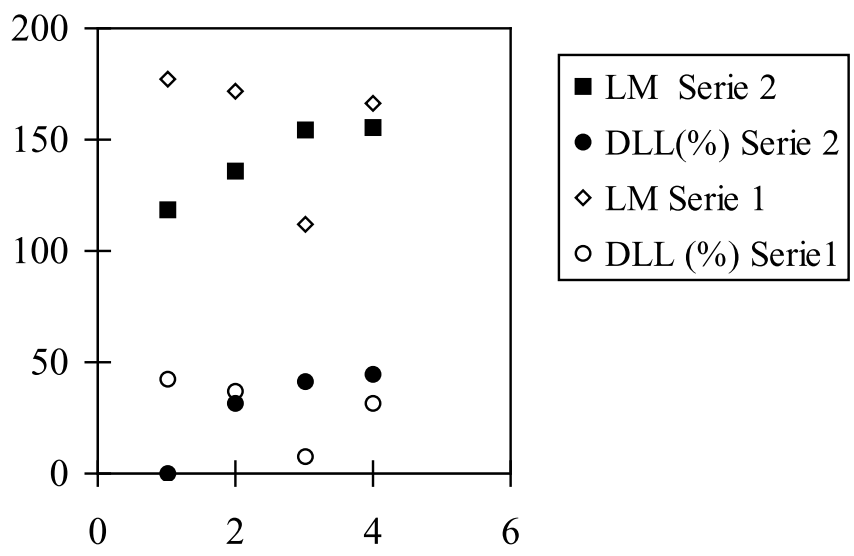

Fig. 8- Tamaños de cristalito promedio (LM, escala en $\AA$ ) de filosilicatos sobrepuestos a valores de disminución del límite líquido (LL), para las series 1 y 2 , de la tabla VIII (muestras $x, 1-1,1-2,1-3,1-4$ y $2-1$, 2-2, 2-3, 2-4, respectivamente). 
TABLA VIII. TAMAÑO APARENTE DE CRISTALITO PROMEDIO Y VARIACIONES DE LÍMITES DE ATTERBERG.ABLA I. ANÁLISIS QUÍMICO. DATOS EXTRAIDOS DE (11)

\begin{tabular}{|c|c|c|c|c|c|c|c|c|c|c|c|c|c|}
\hline$x$ & $\mathrm{~T}\left({ }^{\circ} \mathrm{C}\right)$ & $\mathrm{t}(\mathrm{min})$ & REF. & LL & $\operatorname{DLL}(\%)$ & LP & $\begin{array}{c}\text { DLP } \\
(\%)\end{array}$ & $\mathrm{x}_{1}$ & $\mathrm{x}_{\mathrm{K}}$ & $x_{Q}$ & $\mathrm{Li}$ & Lk & $\begin{array}{c}\mathrm{LM}= \\
\mathrm{x}_{\mathrm{K}} \mathrm{Lk}+\mathrm{x}_{\mathrm{I}} \mathrm{Li}\end{array}$ \\
\hline $1-1$ & 450 & 5 & 13 & 26 & 7.14 & 17.9 & -7.83 & 0.205 & 0.269 & 0.466 & 222.9 & 245.7 & 111.78 \\
\hline $1-2$ & 450 & 5 & 14 & 37 & 42.18 & 27.7 & 4.81 & 0.068 & 0.676 & 0.136 & 164.4 & 245.7 & 177.27 \\
\hline $1-3$ & 450 & 5 & 15 & 32 & 37.25 & 23.8 & 1.65 & 0.089 & 0.638 & 0.055 & 164.4 & 245.7 & 171.38 \\
\hline $1-4$ & 450 & 7 & 16 & 39 & 31.57 & 27.7 & 7.97 & 0.037 & 0.65 & 0.089 & 164.4 & 245.7 & 165.78 \\
\hline $2-1$ & 20 & - & 16 & 67 & - & 30.1 & 0 & 0.037 & 0.65 & 0.089 & 77.09 & 177.9 & 118.48 \\
\hline $2-2$ & 350 & 30 & 16 & 46 & 31.34 & 29.8 & 0.99 & 0.037 & 0.65 & 0.089 & 148.2 & 200.6 & 135.87 \\
\hline $2-3$ & 450 & 5 & 16 & 39 & 41.79 & 27.7 & 7.97 & 0.037 & 0.65 & 0.089 & 164.4 & 227.6 & 154.02 \\
\hline $2-4$ & 450 & 30 & 16 & 37 & 44.77 & 26.9 & 10.61 & 0.037 & 0.65 & 0.089 & 199.2 & 228.3 & 155.76 \\
\hline
\end{tabular}

para cada arcilla de la Tabla V, un tamaño de cristalito promedio LM según:

$\mathrm{LM}=\mathrm{x}_{\mathrm{K}} \mathrm{L}_{\mathrm{K}}+\mathrm{x}_{\mathrm{I}} \mathrm{L}_{\mathrm{I}}$

Donde $x_{K} y x_{I}$ son las fracciones másicas de caolinita e illita (tomadas de la Tabla II) y donde $\mathrm{L}_{K} \mathrm{y} \mathrm{L}_{\mathrm{I}}$ son los tamaños aparentes de cristalito para caolinita e illita respectivamente, obtenidos en experimentos con la muestra 26. Para la muestra PT13 se han utilizado los tamaños de cristalito de la illita de la muestra PT11 (que presenta mayor tamaño de cristalito).

La Fig.8 presenta la evolución de DLL para las muestras PT13, PT14, PT15 y PT16, tras tratamiento térmico a 450 ${ }^{\circ} \mathrm{C}$ durante 5 minutos y sus correspondientes valores de LM (series 1 de DLL y LM), por una parte, y por otra, las series 2 (de DLL y LM) de la muestra 16 con diferentes tratamientos térmicos; en ambos casos las líneas de LM y DLL, siguen la misma pauta de crecimiento o decrecimiento (trazas aproximadamente paralelas).

Para los valores de DLP no se aprecia tan buena correspondencia (no se incluye gráfico), ya que las diferencias son de escasa cuantía, y en algunos casos, próximas a los limites detectables por el procedimiento empleado; con las limitaciones expuestas, la variación observada en PT13 representaría una anomalía ( $\mathrm{DLP}<0$, crecimiento en vez de decrecimiento) interpretable como debida a reabsorción en pequeñas cuantías de láminas de esmectita asociadas a la illita.

Existe una buena correlación $\left(\mathrm{R}^{2}=0.938\right)$ entre DLL en experimentos con la muestra PT16 y los correspondientes a

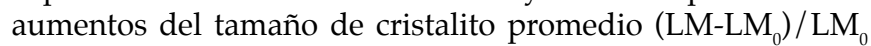
(siendo LM el tamaño medido en la muestra tratada y $\mathrm{LM}_{0^{\prime}}$ en la misma sin tratar). La correlación no es tan buena para el decrecimiento de LP y aumento de LM $\left(R^{2}=0.855\right)$ en estos experimentos, pero se halla en el intervalo de valores de coeficientes de correlación observados entre límites de Atterberg en el estudio de García Tomás y col. (11).

Las imágenes de FESEM en caolinitas (como caso más desfavorable) muestran tamaños de cristalito superiores a los registrados por difracción pero de mismo orden de magnitud, y permiten apreciar que con el tratamiento térmico se produce un aumento de frecuencia de los cristalitos de mayor espesor, así como disminuciones de rugosidad (y en correspondencia, aumentos de compacidad) con la consiguiente disminución del espacio disponible para la adsorción de agua.

\section{CONCLUSIONES}

Se ha podido reconocer una relación entre evolución microestructural de caolinita e illita en las arcillas tratadas y las correspondientes variaciones en los límites de
Atterberg, habiendo resultado útil a tales efectos el análisis microestructural por DRX, dado que existe correlación entre aumentos de tamaño de cristalito y disminuciones de límites de Atterberg.

Parece existir una relación entre el tamaño de cristalito aparente medido por DRX y el tamaño efectivo de las partículas adsorbentes de agua. Ello se ha puesto de manifiesto observando que las arcillas con los tratamientos térmicos aplicados experimentan disminuciones del límite líquido, cuya variación sigue las pautas de variación de los tamaños de cristalito de los filosilicatos arcillosos presentes, ocurriendo que cuando se alcanzan mayores tamaños de cristalito tras el tratamiento, las disminuciones del límite líquido respecto al de la muestra sin tratar son también mayores.

Así, apreciándose un aumento de tamaño de cristalito, dos mecanismos principales de disminución de la superficie efectiva disponible para la interacción con agua pueden invocarse, atendiendo a las observaciones efectuadas en FESEM: a) disminución del número de conjuntos de cristalitos que pueden ser alcanzados como partículas individuales por agua en caras de pinacoides $\{001\}$ y b) decrecimiento de la rugosidad producida por agregados de cristalitos en caras paralelas a [001] o a (001).

\section{AGRADECIMIENTOS}

Este trabajo presenta resultados de los proyectos: .PET 93-100 (Mํo Educación y Ciencia, DGICYT) y GV2004-B-274 (Generalitat Valenciana, CCEC).

\section{BIBLIOGRAFÍA}

1. C.A Jouenne. "Traité des ceramiques et des materiaux mineraux", 1975, Paris, Ed. Septima, 657.

2. E. Bloor. Plasticity : A critical survey. Trans. Brit. Ceram. Soc, 56, 423, (1957)

3. A. Atterberg. Uber die Physikalische Bodenunterschung un über die Plasticitat der Tone. International Mitteilung für Bodenkunde, 1, 10-43, (1911)

4. A. Casagrande. "Research in Atterberg limits of soils". Public Roads, 13, (1932)

5. A. Casagrande. Classification and Identification of Soils. Trans. Am. Soc. Civil Engs, 113, 2351, 901-930, (1948)

6. T.A Klinefelter, H.P. Hamlin. "Syllabus of Clay Testing". Bureau of Mines, Bulletin 565, Washington, U.S.A Government Printing Office, 67. (1957)

7. NERC. "Minerals for Development", BGS ODA Project technical report WG/94/13, (1994)

8. A. Barba, C. Feliu, J. García, F. Ginés, E. Sanchez, V. Sanz, V. Beltrán. "Materias primas para la fabricación de soportes de baldosas cerámicas", ITC-AICE, Castellón. 291. (1997)

9. E. Gippini. “Contribución al conocimiento de la composición óptima de las pastas cerámicas"., Madrid, Patronato Juan De la Cierva, CSIC, 119, (1969) 
10. R.A Grim. "Clay Mineralogy", New York, Mc Graw- Hill Book Cy, 425. (1969)

11. F. García Tomás, J. Bastida, J. de la Torre, V. Esteve, M. Lores, J. Serrano. "Plasticidad y características composicionales de arcillas industriales de la zona minera de Teruel (España) ". Bol. Soc. Esp. Mineralogía, 19, 101-117, (1996)

12. J. Bastida. Procedimiento para la eliminación de la materia orgánica presente en las arcillas cerámicas, y en materiales arcillosos en general. Patente española $\mathrm{N}^{\circ}$ P9601256, de fecha 6-6-96. Titular: Universitat de València, 1996.

13. M.T Lores, S. Font, J. de la Torre, J. Bastida. Estudio de la eliminación de materia orgánica en arcillas mediante tratamiento térmico. Análisis del proceso a diferentes escalas. Bol. Soc. Esp. Ceram. V. 36, [4], 419-424, (1997)

14. D.A. Holderidge, F. Moore. Clay Water Relationship in Ceramics. Clay Min. Bull, 2, 26-33, (1953)

15. H. Salmang. La ceramique au point de vue physico-chimique, Paris, Ed. Dunod, 465, (1935)

16. J.M. Fernández Navarro. La plasticidad de las arcillas. Bol. Soc. Esp. Cerám. y V., 1, [7], 449-473, (1962)

17. P. Ctori. The effects of temperature on the physical properties of cohesive soil. Ground Engineering, 22, [5], 26-27, (1989)

18. N. Deepak. Effect of temperature and pore fluid on shear characteristic of clay. International Syposium on Environmental Geotechnology; Volume 11 Envo Publ. Co., United States, 382-390, (1986)

19. J.G. Laguros. Effect of temperature on some engineering properties of clay soils. Highway Research Board Spec. Rept. 103 (NRC-NAS-NAE, Pub. 1641), (1969)

20. B. Kubler. Les indicateurs de transformations physiques et chimiques dans la diagenèse, temperature et calorimetrie. In "Thermometrie et barométrie geologiques", (M. Lagache Editor), Paris, Soc. Franc. Minér. Crist. 489-596, (1984)

21. M. Frey. Very Low grade metamorphism of clastic sedimentary rocks, 10-58 in "Low Tempearture metamorhism ", (Frey, M, Editor), Glasgow, Blackie, 349. (1987)

22. D. Eberl, J. Srodon, M. Kralik, B.E. Taylor, Z.E. Peterman. Ostwald Ripening of Clays and Metamorphic Minerals. Science, 248, 474-477. (1990)

23. S Guggenheim, D.C Bain,F Bergaya, M.F Brigatti, V.A Drits, M.L.L Formoso, D.D Eberl, E. Galán, R.J Merriman, D.R. Peacor, H. Stanjek, T. Watanabe. Report of the Association Internationale pour l'Etude des Argiles (AIPEA) Nomenclature Committee for 2001: Order, disorder and crystallinity in phyllosilicates and the use of the "crystallinity index". Clays and Clay Minerals 50, [3], 406-409, (2002)

24. J V. Clausell. Análisis microestructural de caolinitas y genésis de caolines en el Macizo Ibérico. Cad. Lab. Xeol. Laxe 26, 11-97, (2001)

25. NLT. "Normas del Laboratorio del Transporte y Mecánica del Suelo". Madrid, Instituto José Luis Escario. (1972)

26. J.M. Amigó, J. Bastida, A. Sanz, M. Signes, J. Serrano. Crystallinity of Lower Cretaceous kaolinites of Teruel (Spain). Applied Clay Science, 9, 1-19, (1994)

27. E.F. Bertaut. Raies de Debye-Scherrer et repartition des dimensions des domaines de Bragg dans les poudres polycrystallines. Acta Cryst. 3, 14-19, (1950)

28. J.I. Langford. A rapid method for analysing the breadths of diffraction and spectral lines using the Voigt function. J. Appl. Cryst. 11, 4-10, (1978).

29. W.A. Deer, R.A. Howie, J. Zussman. An introduction to the rock forming minerals. London, Longman. 526. (1977).

30. M. Zbick, R.S. Smart. Dispersion of kaolinites and Talc in aqueous solution nanomorphology and nanobubble entrapment. Minerals Engineering, 15, 4, 277-286. (2002).

Recibido: 14.02.05

Aceptado: 05.07.05 